\title{
LATTICE-ORDERED GROUPS AND A CONJECTURE FOR ADEQUATE DOMAINS
}

\author{
J. W. BREWER, P. F. CONRAD AND P. R. MONTGOMERY
}

\begin{abstract}
In this paper, we present a counterexample to show that adequate domains are not characterized by the property that nonzero prime ideals are contained in a unique maximal ideal. The counterexample is obtained by constructing a lattice-ordered group with certain properties and exploiting the relation between Bezout domains and their (lattice-ordered) group of divisibility. The domain constructed is an elementary divisor ring with zero Jacobson radical. The lattice-ordered group constructed also shows that various conjectures about $l$-groups are false.
\end{abstract}

In this note we construct a lattice-ordered group which provides not only a counterexample to certain lattice group-theoretic conjectures, but also provides a counterexample to a conjecture in commutative rings. We have arbitrarily chosen to discuss the ring-theoretic conjecture first, saving the group-theoretic considerations until the example has been given.

A problem going back at least as far as [7] is to determine those rings (predictably called elementary divisor rings) for which an elementary divisor theory exists. Even earlier, Helmer [5] showed that not only does the domain of entire functions have such a theory, it also has a property which he labeled adequate. An integral domain $D$ is said to be adequate if $D$ is Bezout (finitely generated ideals of $D$ are principal) and for each $a, b \in D, a \neq 0 \neq b$, there exist $c, d \in D$ such that $a=c d,(b, c)=D$, and if $s$ is a nonunit divisor of $d$, then $(b, s) \neq D$. Henriksen [6] appears to be the first person to have given an example to show that being adequate is a stronger property than that of being an elementary divisor ring. In proving this, he observed that if $D$ is an adequate domain, then each nonzero prime ideal of $D$ is contained in a unique maximal ideal. A natural question to ask is whether or not the converse holds and the question is explicitly raised in [9]. We settle this question in the negative by showing that there exists an elementary divisor ring which is not adequate

Received by the editors May 17, 1973.

AMS (MOS) subject classifications (1970). Primary 06A60, 13A15, 13C05, $13 \mathrm{G} 05$.

Key words and phrases. Lattice ordered abelian group, adequate domain, group of divisibility, elementary divisor ring, lateral completion.

(c) American Mathematical Society 1974 
but which does have the property that each nonzero prime ideal is contained in a unique maximal ideal. Our approach is the by-now standard one of interpreting and solving the problem in the theory of latticeordered abelian groups and then pulling the solution back to integral domains.

Let $D$ be a unitary integral domain with quotient field $K$. We recall several facts. The group of divisibility of $D$, denoted $G(D)$, is the multiplicative group of nonzero principal fractional ideals of $D$, partially ordered by taking as the positive cone the set of principal integral ideals of $D$. If $D$ is a Bezout domain, then the divisibility group of $D$ is a latticeordered group ( $l$-group), and if $J$ is any abelian $l$-group, there exists a Bezout domain $D$ such that $G(D) \cong J$. Moreover, there is a one-to-one inclusion reversing correspondence between the set of prime ideals of a Bezout domain and the set of prime subgroups of its divisibility group. (A convex $l$-subgroup $S$ of an $l$-group $G$ is prime if $G / S$ is totally ordered, or equivalently, if $a \wedge b=0$ in $G$, then $a \in S$ or $b \in S$.) The reader who desires more details is referred to [4, Chapter III].

If $D$ is a Bezout domain, the adequate condition on $D$ translates readily into a condition on $G(D)$ ([9]), and conversely, if $G(D)$ satisfies this condition, then $D$ must be adequate. Theorem 4.7 of [9] shows that $D$ is adequate if and only if (in the terminology of $l$-groups) $G(D)$ is projectable. An l-group is projectable if each principal polar is a cardinal summand; that is, $G=g^{\prime \prime} \boxplus g^{\prime}$ for each $g \in G$, where $g^{\prime}=\{x \in G|| x \mid \wedge$ $|g|=0\}, g^{\prime \prime}=\left\{y \in G|| x|\wedge| y \mid=0\right.$ for each $\left.x \in g^{\prime}\right\}$.

The above remarks show that our problem is to find an abelian l-group $G$ that satisfies: (1) Each proper prime subgroup of $G$ contains a unique minimal prime subgroup, but not: (2) $G$ is projectable.

Consider the following partially ordered set

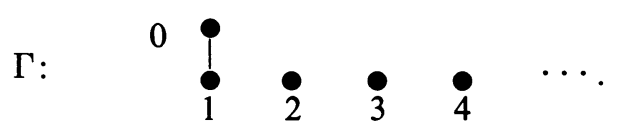

Let $V$ be the set of all integer valued functions on $\Gamma$ and define $v=\left(v_{0}, v_{1}, v_{2}, \cdots\right)$ to be positive if $v_{0}>0$ and $v_{i} \geqq 0, i=2,3, \cdots$, or $v_{0}=0$ and $v_{i} \geqq 0$ for $i=1,2,3, \cdots$. Then $V$ is an $l$-group. Let $G$ be the subgroup of $V$ generated by the small or restricted direct sum on $1,2,3, \cdots$, and the element $a=(1,0,1,0,1,0, \cdots)$. It is easy to check that if $g \in G$, then $g \vee 0 \in G$ and hence $G$ is an $l$-subgroup of $V$.

Let $v(i)$ be the characteristic function on $i$ for $i=1,2,3, \cdots$. Then $G=v(i)^{\prime \prime} \boxplus v(i)^{\prime}$ for $i=2,3, \cdots$, but $a \in G \backslash\left(v(1)^{\prime \prime} \boxplus v(1)^{\prime}\right)$ so that $G$ does not satisfy (2). 
Let $U_{0}=\left\{v \in G \mid v_{0}=0\right\}, \quad U_{1}=\left\{v \in G \mid v_{0}=v_{1}=0\right\}$, and for $i=2,3, \cdots$, $U_{i}=\left\{v \in G \mid v_{i}=0\right\}$.

It is easy to check that $G / U_{j}$ is totally ordered, so that each $U_{j}$ is a proper prime subgroup of $G$. Suppose that $P$ is a prime subgroup of $G$. If $v(i) \in P$ for $i=2,3, \cdots$, then $P \supseteq U_{1}$, and hence $P=U_{0}$ or $P=U_{1}$. If $v(j) \notin P$ for some $j>1$, then $U_{j}=v(j)^{\prime} \subseteq P$ and hence $P=U_{j}$. Thus, $\left\{U_{i}\right\}_{0}^{\infty}$ is the set of all proper prime subgroups of $G$ and hence $G$ satisfies (1).

Let $D$ be a Bezout domain whose group of divisibility is isomorphic to $G$. We remark that $D$ must be an elementary divisor ring; $D$ has only countably many maximal ideals and as Kaplansky notes in [8, p. 80], such a Bezout domain must be an elementary divisor ring. Moreover it is easy to see that $G$ is the union of its minimal primes and thus $D$ has Jacobson radical (0). The aforementioned example of Henriksen has Jacobson radical $\neq(0)$.

In summary, the domain $D$, whose group of divisibility is $G$, has the following properties:

(a) $D$ is an elementary divisor domain.

(b) Each nonzero prime ideal of $D$ is contained in a unique maximal ideal.

(c) $D$ is not adequate.

(d) $D$ has Jacobson radical $=(0)$.

Turning now to l-groups, it is interesting to trace the relationship between (1) and (2) in l-groups. Bigard [1] derives a condition that is necessary and sufficient for (2) and states that this condition is stronger than (1). However, he gives no example to substantiate this claim. The question whether (1) implies (2) was posed in [3].

A value of an element $d$ in an $l$-group $A$ is a convex $l$-subgroup $M$ of $A$ that is maximal with respect to not containing $d$. $A$ is finite-valued if each $0 \neq d \in A$ has only a finite number of values. If $A$ is finite-valued, then (1) implies (2). For rings, the analogous result is also known-namely, if $D$ is a Bezout domain such that each nonzero prime ideal is contained in a unique maximal ideal, and if each nonzero element of $D$ is contained in only finitely many maximal ideals, then $D$ is adequate [9, Theorem 4.3].

A convex $l$-subgroup $C$ of an $l$-group $A$ is closed if for $\left\{a_{\lambda} \mid \lambda \in \Lambda\right\} \subseteq C$ and if $a=\bigvee a_{\lambda}$ exists, then $a \in C$. We see that:

Each prime subgroup of $G$ is closed.

Thus, (3) does not imply that $G$ is finite-valued. This settles an open question in $l$-groups that has been around for some years. It is known that if $A$ is finite-valued, then $A$ satisfies (3), and that (3), in turn, is equivalent to quite a few interesting properties of $A$. 
An l-group is laterally complete if each disjoint subset has a least upper bound. One may verify that $V$ is the lateral completion of $G$; that is, $V$ is laterally complete, no proper $l$-subgroup of $V$ containing $G$ is laterally complete, and each positive element in $V$ exceeds a positive element in $G$.

An $a^{*}$-extension of an abelian $l$-group $A$ is an abelian $l$-group $H$ that contains $A$ as an $l$-subgroup and is such that the correspondence $K \rightarrow K \cap A$ is a one-to-one map of the set of closed convex $l$-subgroups of $H$ onto the set of closed convex $l$-subgroups of $G$.

In [2], it is shown that each abelian $l$-group admits an $a^{*}$-closure (an $a^{*}$-extension that admits no proper $a^{*}$-extension). An open question from [2] is whether or not an $a^{*}$-closure contains the lateral completion. The answer is no since $V$ is not an $a^{*}$-extension of $G$. For, if $B=\{v \in$ $\left.V \mid v_{i}=0, i=2,3, \cdots\right\}, C=\left\{v \in V \mid v_{i}=0, i=0,2,3, \cdots\right\}$, then $B$ and $C$ are closed convex $l$-subgroups of $V$, but $B \cap G=C \cap G=C$.

Hence, the $l$-group $V$ and its $l$-subgroup $G$ satisfy the following:

(1) Each prime subgroup of $G$ contains a unique minimal prime subgroup.

(2) $G$ is not projectable.

(3) Each prime subgroup of $G$ is closed.

(4) $G$ is not finite-valued.

(5) $V$ is the lateral completion of $G$, but $V$ is not an $a^{*}$-extension of $G$.

This example can also be used to show that a polar $C$ of an $a^{*}$-closed l-group $H$ need not be $a^{*}$-closed and $H / C$ need not be $a^{*}$-closed. Here $H$ is the $a^{*}$-closure of $G$.

In [2] there is an example of an l-group that satisfies (1) through (4), but it is much more complicated than the one given here. Richard Byrd has sent us an example, similar to the one in [2], that satisfies (1) through (4). Our example is an l-homomorphic image of Byrd's. He also has a method for constructing $l$-groups that satisfy (3) and (4).

\section{BIBLIOGRAPHY}

1. A. Bigard, Contribution à la théorie des groupes réticulés, Thèse, Université Paris, 1969.

2. R. Bleier and P. Conrad, The lattice of closed ideals and $a^{*}$-extensions of an abelian l-group, Pacific J. Math. (to appear).

3. P. Conrad and D. McAlister, The completion of a lattice-ordered group, J. Austral. Math. Soc. 9 (1969), 182-208. MR 40 \#2585.

4. R. Gilmer, Multiplicative ideal theory, Dekker, New York, 1972.

5. $\mathrm{O}$. Helmer, The elementary divisor theorem for certain rings without chain conditions, Bull. Amer. Math. Soc. 49 (1943), 225-236. MR 4, 185.

6. M. Henriksen, Some remarks on elementary divisor rings. II, Michigan Math. J. $3(1955 / 56), 159-163$. MR 19, 1155. 
7. I. Kaplansky, Elementary divisors and modules, Trans. Amer. Math. Soc. 66 (1949), 464-491. MR 11, 155.

8. - Infinite abelian groups, rev. ed., Univ. of Michigan Press, Ann Arbor, Mich., 1969. MR 38 \#2208.

9. M. Larsen, W. Lewis and T. Shores, Elementary divisor rings and finitely presented modules, Trans. Amer. Math. Soc. (to appear).

Department of Mathematics, University of Kansas, Lawrence, Kansas 66044 